\title{
On the joint spectral radius
}

\author{
by Vladimír Müller (Praha)
}

Abstract. We prove the $\ell_{p}$-spectral radius formula for $n$-tuples of commuting Banach algebra elements. This generalizes results of some earlier papers.

Let $A$ be a Banach algebra with the unit element denoted by 1 . Let $a=\left(a_{1}, \ldots, a_{n}\right)$ be an $n$-tuple of elements of $A$. Denote by $\sigma(a)$ the Harte spectrum of $a$, i.e. $\lambda=\left(\lambda_{1}, \ldots, \lambda_{n}\right) \notin \sigma(a)$ if and only if there exist $u_{1}, \ldots, u_{n}, v_{1}, \ldots, v_{n} \in A$ such that

$$
\sum_{j=1}^{n}\left(a_{j}-\lambda_{j}\right) u_{j}=\sum_{j=1}^{n} v_{j}\left(a_{j}-\lambda_{j}\right)=1 .
$$

Let $1 \leq p \leq \infty$. The (geometric) spectral radius of $a$ is defined by

$$
r_{p}(a)=\max \left\{\|\lambda\|_{p}: \lambda \in \sigma(a)\right\}
$$

where

$$
\|\lambda\|_{p}= \begin{cases}\max _{1 \leq j \leq n}\left|\lambda_{j}\right| & (p=\infty), \\ \left(\sum_{j=1}^{n}\left|\lambda_{j}\right|^{p}\right)^{1 / p} & (1 \leq p<\infty) ;\end{cases}
$$

see [10], cf. also [4].

If $\sigma(a)$ is empty we put formally $r_{p}(a)=-\infty$.

Clearly, $r_{p}(a)$ depends on $p$. On the other hand, instead of the Harte spectrum we can take any other reasonable spectrum (e.g. the left, right, approximate point, defect, Taylor etc.) without changing the value of $r_{p}(a)$; see $[4],[9]$.

For a single Banach algebra element the just defined spectral radius $r_{p}(a)$ does not depend on $p$ and coincides with the ordinary spectral radius $r\left(a_{1}\right)=\max \left\{\left|\lambda_{1}\right|: \lambda_{1} \in \sigma\left(a_{1}\right)\right\}$. By the well-known spectral radius formula

1991 Mathematics Subject Classification: Primary 46H05, 46J05.

Key words and phrases: Banach algebra, spectrum, spectral radius.

The research was supported by the grant No. 119106 of the Academy of Sciences of the Czech Republic. 
we have in this case

$$
r\left(a_{1}\right)=\lim _{k \rightarrow \infty}\left\|a_{1}^{k}\right\|^{1 / k}=\inf _{k}\left\|a_{1}^{k}\right\|^{1 / k} .
$$

The spectral radius formula for $n$-tuples of Banach algebra elements was studied by a number of authors, see e.g. [1], [2], [6], [7], [8]. In this paper we generalize results of [6], [7] and [10].

Let $a=\left(a_{1}, \ldots, a_{n}\right)$ be an $n$-tuple of elements of a Banach algebra $A$. Instead of powers of a single element it is natural to consider all possible products of $a_{1}, \ldots, a_{n}$.

Denote by $F(k, n)$ the set of all functions from $\{1, \ldots, k\}$ to $\{1, \ldots, n\}$. Let further

$$
s_{k, p}(a)=\left(\sum_{f \in F(k, n)}\left\|a_{f(1)} \ldots a_{f(k)}\right\|^{p}\right)^{1 / p} \quad(1 \leq p<\infty)
$$

and

$$
s_{k, \infty}(a)=\max _{f \in F(k, n)}\left\|a_{f(1)} \ldots a_{f(k)}\right\| .
$$

LEMMA 1. $s_{k+l, p} \leq s_{k, p}(a) \cdot s_{l, p}(a)$

Proof. The statement is obvious for $p=\infty$. For $p<\infty$ we have

$$
\begin{aligned}
{\left[s_{k, p}(a) \cdot s_{l, p}(a)\right]^{p} } & =\sum_{f \in F(k, n)}\left\|a_{f(1)} \ldots a_{f(k)}\right\|^{p} \cdot \sum_{g \in F(l, n)}\left\|a_{g(1)} \ldots a_{g(l)}\right\|^{p} \\
& \geq \sum_{f, g}\left\|a_{f(1)} \ldots a_{f(k)} a_{g(1)} \ldots a_{g(l)}\right\|^{p}=\left[s_{k+l, p}(a)\right]^{p} .
\end{aligned}
$$

It is well known that the above lemma implies that $\lim _{k \rightarrow \infty}\left(s_{k, p}(a)\right)^{1 / k}$ exists and it is equal to $\inf _{k}\left(s_{k, p}(a)\right)^{1 / k}$.

Thus we may define

$$
r_{p}^{\prime \prime}(a)=\lim _{k \rightarrow \infty}\left(\sum_{f \in F(k, n)}\left\|a_{f(1)} \ldots a_{f(k)}\right\|^{p}\right)^{1 /(p k)} .
$$

Similarly we define

$$
r_{p}^{\prime}(a)=\limsup _{k \rightarrow \infty}\left(\sum_{f \in F(k, n)} r^{p}\left(a_{f(1)} \ldots a_{f(k)}\right)\right)^{1 /(p k)}
$$

(we write briefly $r^{p}(x)$ instead of $(r(x))^{p}$ ).

In general, the limit in (1) does not exist. The limit exists if $a_{1}, \ldots, a_{n}$ are mutually commuting. This can be proved analogously as in Lemma 1 by using the submultiplicativity of the spectral radius.

THEOREM 2. Let $a=\left(a_{1}, \ldots, a_{n}\right)$ be an $n$-tuple of elements of a Banach algebra $A$. Let $1 \leq p \leq \infty$. Then

$$
r_{p}(a) \leq r_{p}^{\prime}(a) \leq r_{p}^{\prime \prime}(a)
$$


Proof. The case $p=\infty$ was proved in [7], Theorem 1 .

Let $p<\infty$. The second inequality is clear.

Let $\lambda=\left(\lambda_{1}, \ldots, \lambda_{n}\right) \in \sigma(a)$. Denote by $A_{0}$ the closed subalgebra of $A$ generated by the unit 1 and the elements $a_{1}, \ldots, a_{n}$. By [5], Proposition 2, there exists a multiplicative functional $h: A_{0} \rightarrow \mathbb{C}$ such that $h\left(a_{j}\right)=\lambda_{j}$ for $j=1, \ldots, n$. Then

$$
\begin{aligned}
\sum_{f \in F(k, n)} r^{p}\left(a_{f(1)} \ldots a_{f(k)}\right) & \geq \sum_{f \in F(k, n)}\left|h\left(a_{f(1)} \ldots a_{f(k)}\right)\right|^{p} \\
& =\sum_{f \in F(k, n)}\left|\lambda_{f(1)}\right|^{p} \ldots\left|\lambda_{f(k)}\right|^{p} \\
& =\left(\left|\lambda_{1}\right|^{p}+\ldots+\left|\lambda_{n}\right|^{p}\right)^{k}=\|\lambda\|_{p}^{p k} .
\end{aligned}
$$

Thus

and $r_{p}^{\prime}(a) \geq r_{p}(a)$.

$$
\sum_{f \in F(k, n)} r^{p}\left(a_{f(1)} \ldots a_{f(k)}\right) \geq r_{p}^{p k}(a)
$$

If $a=\left(a_{1}, \ldots, a_{n}\right)$ is an $n$-tuple of mutually commuting elements then a better result can be proved.

We use the standard multiindex notation. Denote by $\mathbb{Z}_{+}$the set of all non-negative integers. For $\alpha=\left(\alpha_{1}, \ldots, \alpha_{n}\right) \in \mathbb{Z}_{+}^{n}$ and $m \in \mathbb{Z}_{+}$define $|\alpha|=$ $\alpha_{1}+\ldots+\alpha_{n}, \alpha !=\alpha_{1} ! \ldots \alpha_{n} !, a^{\alpha}=a_{1}^{\alpha_{1}} \ldots a_{n}^{\alpha_{n}}$ and $m \alpha=\left(m \alpha_{1}, \ldots, m \alpha_{n}\right)$. If $k$ is an integer, $k \geq|\alpha|$, then let

$$
\left(\begin{array}{l}
k \\
\alpha
\end{array}\right)=\frac{k !}{\alpha !(k-|\alpha|) !}
$$

(for $n=1$ this definition coincides with the classical binomial coefficients).

We shall use frequently the following formula (for commuting variables $\left.x_{i}\right)$ :

$$
\left(x_{1}+\ldots+x_{n}\right)^{k}=\sum_{|\alpha|=k}\left(\begin{array}{l}
k \\
\alpha
\end{array}\right) x^{\alpha} .
$$

In particular, for $x_{1}=\ldots=x_{n}=1$ we have $\sum_{|\alpha|=k}\left(\begin{array}{l}k \\ \alpha\end{array}\right)=n^{k}$.

If $a=\left(a_{1}, \ldots, a_{n}\right)$ is a commuting $n$-tuple of elements of a Banach algebra $A$, then the definitions of $r_{p}^{\prime}(a)$ and $r_{p}^{\prime \prime}(a)$ assume a simpler form (for $1 \leq p<\infty$ ):

$$
\begin{aligned}
& r_{p}^{\prime}(a)=\lim _{k \rightarrow \infty}\left[\sum_{|\alpha|=k}\left(\begin{array}{l}
k \\
\alpha
\end{array}\right) r^{p}\left(a^{\alpha}\right)\right]^{1 /(p k)}, \\
& r_{p}^{\prime \prime}(a)=\lim _{k \rightarrow \infty}\left[\sum_{|\alpha|=k}\left(\begin{array}{l}
k \\
\alpha
\end{array}\right)\left\|a^{\alpha}\right\|^{p}\right]^{1 /(p k)} .
\end{aligned}
$$


TheOREM 3. Let $a=\left(a_{1}, \ldots, a_{n}\right)$ be an $n$-tuple of mutually commuting elements of a Banach algebra $A$. Let $1 \leq p \leq \infty$. Then

$$
r_{p}(a)=r_{p}^{\prime}(a)=r_{p}^{\prime \prime}(a) .
$$

Proof. For $p=\infty$ the first equality was proved in [10] and the second in [7], Theorem 2.

We assume in the following $p<\infty$.

Recall that the number of all partitions of the set $\{1, \ldots, k\}$ into $n$ parts is equal to $\left(\begin{array}{c}k+n-1 \\ n-1\end{array}\right) \leq(k+n-1)^{n-1}$.

We have

$$
\max _{|\alpha|=k}\left(\begin{array}{c}
k \\
\alpha
\end{array}\right)\left\|a^{\alpha}\right\|^{p} \leq \sum_{|\alpha|=k}\left(\begin{array}{l}
k \\
\alpha
\end{array}\right)\left\|a^{\alpha}\right\|^{p} \leq\left(\begin{array}{c}
k+n-1 \\
n-1
\end{array}\right) \max _{|\alpha|=k}\left(\begin{array}{l}
k \\
\alpha
\end{array}\right)\left\|a^{\alpha}\right\|^{p} .
$$

Note that

$$
\lim _{k \rightarrow \infty}\left(\begin{array}{c}
k+n-1 \\
n-1
\end{array}\right)^{1 / k}=1
$$

Thus

$$
r_{p}^{\prime \prime}(a)=\lim _{k \rightarrow \infty}\left[\sum_{|\alpha|=k}\left(\begin{array}{c}
k \\
\alpha
\end{array}\right)\left\|a^{\alpha}\right\|^{p}\right]^{1 /(k p)}=\lim _{k \rightarrow \infty} \max _{|\alpha|=k}\left[\left(\begin{array}{c}
k \\
\alpha
\end{array}\right)\left\|a^{\alpha}\right\|^{p}\right]^{1 /(k p)} .
$$

Similarly,

$$
r_{p}^{\prime}(a)=\lim _{k \rightarrow \infty} \max _{|\alpha|=k}\left[\left(\begin{array}{l}
k \\
\alpha
\end{array}\right) r^{p}\left(a^{\alpha}\right)\right]^{1 /(k p)} .
$$

We now prove the inequality $r_{p}^{\prime}(a) \leq r_{p}(a)$ :

Choose $k$ and $\alpha \in \mathbb{Z}_{+}^{n},|\alpha|=k$. Let $\mu \in \sigma\left(a^{\alpha}\right)$ satisfy $|\mu|=r\left(a^{\alpha}\right)$. By the spectral mapping property there exists $\lambda=\left(\lambda_{1}, \ldots, \lambda_{n}\right) \in \sigma(a)$ such that $\mu=\lambda_{1}^{\alpha_{1}} \ldots \lambda_{n}^{\alpha_{n}}$. Then

$$
\begin{aligned}
\left(\begin{array}{c}
k \\
\alpha
\end{array}\right) r_{p}^{p}\left(a^{\alpha}\right) & =\left(\begin{array}{c}
k \\
\alpha
\end{array}\right)|\mu|^{p}=\left(\begin{array}{c}
k \\
\alpha
\end{array}\right)\left|\lambda_{1}\right|^{\alpha_{1} p} \ldots\left|\lambda_{n}\right|^{\alpha_{n} p} \\
& \leq \sum_{|\beta|=k}\left(\begin{array}{c}
k \\
\beta
\end{array}\right)\left|\lambda_{1}\right|^{\beta_{1} p} \ldots\left|\lambda_{n}\right|^{\beta_{n} p} \\
& =\left(\left|\lambda_{1}\right|^{p}+\ldots+\left|\lambda_{n}\right|^{p}\right)^{k}=\|\lambda\|_{p}^{p k} \leq r_{p}^{p k}(a) .
\end{aligned}
$$

Thus

$$
r_{p}^{\prime}(a)=\lim _{k \rightarrow \infty} \max _{|\alpha|=k}\left[\left(\begin{array}{l}
k \\
\alpha
\end{array}\right) r^{p}\left(a^{\alpha}\right)\right]^{1 /(k p)} \leq r_{p}(a) .
$$

The remaining inequality $r_{p}^{\prime \prime}(a) \leq r_{p}^{\prime}(a)$ will be proved by induction on $n$.

For $n=1$, Theorem 3 reduces to the well-known spectral radius formula for a single element. 
Let $n \geq 2$ and suppose that the inequality $r_{p}^{\prime \prime} \leq r_{p}^{\prime}$ is true for all commuting $(n-1)$-tuples.

For each $k$ there is $\alpha \in \mathbb{Z}_{+}^{n},|\alpha|=k$, such that

$$
\left(\begin{array}{c}
k \\
\alpha
\end{array}\right)\left\|a^{\alpha}\right\|^{p}=\max _{|\beta|=k}\left(\begin{array}{c}
k \\
\beta
\end{array}\right)\left\|a^{\beta}\right\|^{p} .
$$

Using the compactness of $[0,1]^{n}$ we can choose a sequence

$$
\{\alpha(i)\}_{i=1}^{\infty}=\left\{\left(\alpha_{1}(i), \ldots, \alpha_{n}(i)\right)\right\}_{i=1}^{\infty} \subset \mathbb{Z}_{+}^{n}
$$

such that $\lim _{i \rightarrow \infty}|\alpha(i)|=\infty$,

$$
\left(\begin{array}{c}
|\alpha(i)| \\
\alpha(i)
\end{array}\right)\left\|a^{\alpha(i)}\right\|^{p}=\max _{|\beta|=|\alpha(i)|}\left(\begin{array}{c}
|\alpha(i)| \\
\beta
\end{array}\right)\left\|a^{\beta}\right\|^{p} \quad(i=1,2, \ldots)
$$

and the sequences $\left\{\alpha_{j}(i) /|\alpha(i)|\right\}_{i=1}^{\infty}$ are convergent for $j=1, \ldots, n$. Define $k(i)=|\alpha(i)|$ and

$$
t_{j}=\lim _{i \rightarrow \infty} \frac{\alpha_{j}(i)}{k(i)} \in[0,1] \quad(j=1, \ldots, n) .
$$

By (2) we have

$$
r_{p}^{\prime \prime p}(a)=\lim _{i \rightarrow \infty}\left[\left(\begin{array}{c}
k(i) \\
\alpha(i)
\end{array}\right)\left\|a^{\alpha(i)}\right\|^{p}\right]^{1 /(k(i) p)} .
$$

We distinguish two cases:

(a) $t_{j}=0$ for some $j, 1 \leq j \leq n$. Without loss of generality we may assume that $t_{n}=0$. Define $a^{\prime}=\left(a_{1}, \ldots, a_{n-1}\right), \alpha^{\prime}(i)=\left(\alpha_{1}(i), \ldots, \alpha_{n-1}(i)\right)$ $\in \mathbb{Z}_{+}^{n-1}$ and $k^{\prime}(i)=\left|\alpha^{\prime}(i)\right|=k(i)-\alpha_{n}(i)$. Clearly $\lim _{i \rightarrow \infty} k^{\prime}(i) / k(i)=1$. We have $\left\|a^{\alpha(i)}\right\| \leq\left\|a^{\prime \alpha^{\prime}(i)}\right\| \cdot\left\|a_{n}\right\|^{\alpha_{n}(i)}$. Then

$$
r_{p}^{\prime \prime p}\left(a^{\prime}\right) \geq \limsup _{i \rightarrow \infty}\left[\left(\begin{array}{l}
k^{\prime}(i) \\
\alpha^{\prime}(i)
\end{array}\right)\left\|a^{\prime \alpha^{\prime}(i)}\right\|^{p}\right]^{1 / k^{\prime}(i)} \geq L_{1} \cdot L_{2} \cdot L_{3},
$$

where

and

$$
\begin{aligned}
& L_{1}=\limsup _{i \rightarrow \infty}\left[\left(\begin{array}{c}
k^{\prime}(i) \\
\alpha^{\prime}(i)
\end{array}\right) /\left(\begin{array}{c}
k(i) \\
\alpha(i)
\end{array}\right)\right]^{1 / k^{\prime}(i)}, \\
& L_{2}=\lim _{i \rightarrow \infty}\left[\left(\begin{array}{c}
k(i) \\
\alpha(i)
\end{array}\right)\left\|a^{\alpha(i)}\right\|^{p}\right]^{1 / k^{\prime}(i)}
\end{aligned}
$$

$$
L_{3}=\lim _{i \rightarrow \infty}\left\|a_{n}\right\|^{-\alpha_{n}(i) p / k^{\prime}(i)} .
$$

Since $\lim _{i \rightarrow \infty} \alpha_{n}(i) / k^{\prime}(i)=0$, we have $L_{3}=1$. 
Further,

$$
L_{2}=\lim _{i \rightarrow \infty}\left[\left[\left(\begin{array}{c}
k(i) \\
\alpha(i)
\end{array}\right)\left\|a^{\alpha(i)}\right\|^{p}\right]^{1 / k(i)}\right]^{k(i) / k^{\prime}(i)}=r_{p}^{\prime \prime p}(a) .
$$

Finally,

$$
\begin{aligned}
L_{1} & =\limsup _{i \rightarrow \infty}\left[\frac{k^{\prime}(i) ! \cdot \alpha_{n}(i) !}{k(i) !}\right]^{1 / k^{\prime}(i)} \geq \limsup _{i \rightarrow \infty}\left[\frac{\left(\alpha_{n}(i) / 3\right)^{\alpha_{n}(i)}}{k(i)^{\alpha_{n}(i)}}\right]^{1 / k^{\prime}(i)} \\
& =\limsup _{i \rightarrow \infty}\left(\frac{\alpha_{n}(i)}{3 k(i)}\right)^{\left(\alpha_{n}(i) / k(i)\right) \cdot\left(k(i) / k^{\prime}(i)\right)}=1
\end{aligned}
$$

since $\lim _{i \rightarrow \infty} k(i) / k^{\prime}(i)=1$ and

$$
\lim _{i \rightarrow \infty}\left(\frac{\alpha_{n}(i)}{3 k(i)}\right)^{\alpha_{n}(i) / k(i)}=\lim _{x \rightarrow 0_{+}}\left(\frac{x}{3}\right)^{x}=\lim _{x \rightarrow 0_{+}} x^{x}=\lim _{x \rightarrow 0_{+}} e^{x \ln x}=1 .
$$

Thus $r_{p}^{\prime \prime}\left(a^{\prime}\right) \geq r_{p}^{\prime \prime}(a)$.

By the induction assumption $r_{p}^{\prime \prime}\left(a^{\prime}\right)=r_{p}^{\prime}\left(a^{\prime}\right)=r_{p}\left(a^{\prime}\right)$ and by the definition $r_{p}\left(a^{\prime}\right) \leq r_{p}(a)=r_{p}^{\prime}(a)$. Hence $r_{p}^{\prime \prime}(a) \leq r_{p}^{\prime}(a)$.

(b) There remains the case $t_{j}>0(j=1, \ldots, n)$, with $t_{j}=$ $\lim _{i \rightarrow \infty} \alpha_{j}(i) / k(i)$. Choose $\varepsilon>0, \varepsilon<\min _{1 \leq j \leq n} t_{j} / n$. For $i$ sufficiently large we have

$$
t_{j}-\frac{\varepsilon}{4} \leq \frac{\alpha_{j}(i)}{k(i)} \leq t_{j}+\frac{\varepsilon}{4} .
$$

We approximate $t_{1}, \ldots, t_{n}$ by rational numbers. Fix positive integers $c_{1}, \ldots, c_{n}, d$ such that

$$
t_{j}-\frac{\varepsilon}{2} \leq \frac{c_{j}}{d} \leq t_{j}-\frac{\varepsilon}{4} \quad(j=1, \ldots, n)
$$

Let $\gamma=\left(c_{1}, \ldots, c_{n}\right) \in \mathbb{Z}_{+}^{n}$ and $u=a^{\gamma}=a_{1}^{c_{1}} \ldots a_{n}^{c_{n}}$. For each $i$ write $k(i)=m(i) d+z(i)$, where $0 \leq z(i) \leq d-1$. So, for $i$ sufficiently large, we have

and

$$
\frac{c_{j}}{d} \leq \frac{\alpha_{j}(i)}{k(i)}, \quad \frac{\alpha_{j}(i)}{k(i)}-\frac{c_{j}}{d} \leq \frac{3 \varepsilon}{4}
$$

$$
\alpha_{j}(i)-m(i) c_{j}=\alpha_{j}(i)-\frac{k(i)-z(i)}{d} \cdot c_{j}=k(i)\left[\frac{\alpha_{j}(i)}{k(i)}-\frac{c_{j}}{d}\right]+\frac{z(i) c_{j}}{d} .
$$

Thus $\alpha_{j}(i)-m(i) c_{j} \geq 0(1 \leq j \leq n)$ and

$$
k(i)-m(i)|\gamma|=\sum_{j=1}^{n}\left(\alpha_{j}(i)-m(i) c_{j}\right) \leq k(i) \cdot \frac{3 \varepsilon n}{4}+\sum_{j=1}^{n} \frac{z(i) c_{j}}{d} \leq \varepsilon n k(i)
$$

for $i$ large enough. We have 


$$
\begin{aligned}
\left\|a^{\alpha(i)}\right\| & \leq\left\|a_{1}^{m(i) c_{1}} \ldots a_{n}^{m(i) c_{n}}\right\| \cdot\left\|a_{1}\right\|^{\alpha_{1}(i)-m(i) c_{1}} \ldots\left\|a_{n}\right\|^{\alpha_{n}(i)-m(i) c_{n}} \\
& \leq\left\|u^{m(i)}\right\| \cdot K^{n \varepsilon k(i)},
\end{aligned}
$$

where $K=\max \left\{1,\left\|a_{1}\right\|, \ldots,\left\|a_{n}\right\|\right\}$. Then, since $\left(\begin{array}{c}m(i)|\gamma| \\ m(i) \gamma\end{array}\right)^{1 /(m(i)|\gamma|)} \leq n$, we have

$$
\begin{aligned}
r_{p}^{\prime p}(a) & \geq \limsup _{i \rightarrow \infty}\left[\left(\begin{array}{c}
m(i)|\gamma| \\
m(i) \gamma
\end{array}\right) r^{p}\left(a^{m(i) \gamma}\right)\right]^{1 /(m(i)|\gamma|)} \\
& =\limsup _{i \rightarrow \infty}\left(\begin{array}{c}
m(i)|\gamma| \\
m(i) \gamma
\end{array}\right) \cdot r(m)^{1 /(i)|\gamma|)} \cdot r(u)^{p /|\gamma|} \\
& =\limsup _{i \rightarrow \infty}\left[\left(\begin{array}{c}
m(i)|\gamma| \\
m(i) \gamma
\end{array}\right)\left\|u^{m(i)}\right\|^{p}\right]^{1 /(m(i)|\gamma|)} \geq L_{1} \cdot L_{2} \cdot L_{3},
\end{aligned}
$$

where

and

$$
\begin{aligned}
& L_{1}=\liminf _{i \rightarrow \infty}\left[\left(\begin{array}{c}
m(i)|\gamma| \\
m(i) \gamma
\end{array}\right) /\left(\begin{array}{c}
k(i) \\
\alpha(i)
\end{array}\right)\right]^{1 /(m(i)|\gamma|)}, \\
& L_{2}=\liminf _{i \rightarrow \infty}\left[\left(\begin{array}{c}
k(i) \\
\alpha(i)
\end{array}\right)\left\|a^{\alpha(i)}\right\|^{p}\right]^{1 /(m(i)|\gamma|)}
\end{aligned}
$$

$$
L_{3}=\liminf _{i \rightarrow \infty} K^{-n \varepsilon p k(i) /(m(i)|\gamma|)}
$$

Since

$$
1 \leq \frac{k(i)}{m(i)|\gamma|} \leq \frac{1}{1-n \varepsilon}
$$

for $i$ sufficiently large, we have $L_{3} \geq K^{-n \varepsilon p /(1-n \varepsilon)}$.

Since

$$
\lim _{i \rightarrow \infty}\left[\left(\begin{array}{c}
k(i) \\
\alpha(i)
\end{array}\right)\left\|a^{\alpha(i)}\right\|^{p}\right]^{1 / k(i)}=r_{p}^{\prime \prime p}(a),
$$

we have $L_{2} \geq \min \left\{r_{p}^{\prime \prime p}(a),\left(r_{p}^{\prime \prime p}(a)\right)^{1 /(1-n \varepsilon)}\right\}$.

To estimate $L_{1}$, we use the well-known Stirling formula

$$
l !=l^{l} e^{-l} \sqrt{2 \pi l}(1+o(l)) .
$$

We have

$$
\begin{aligned}
(1-\varepsilon)\left(\frac{\alpha_{j}(i)}{e}\right)^{\alpha_{j}(i) /(m(i)|\gamma|)} & \leq\left(\alpha_{j}(i) !\right)^{1 /(m(i)|\gamma|)} \\
& \leq(1+\varepsilon)\left(\frac{\alpha_{j}(i)}{e}\right)^{\alpha_{j}(i) /(m(i)|\gamma|)}
\end{aligned}
$$

for $j=1, \ldots, n$ and for $i$ sufficiently large. Similar estimates can be used for $\left(m(i) c_{j}\right) !,(m(i)|\gamma|)$ ! and $|\alpha(i)| !$. Thus, for $i$ sufficiently large, we have (to 
simplify the expressions we write $m, k$ and $\alpha$ instead of $m(i), k(i)$ and $\alpha(i))$

$$
\begin{aligned}
& {\left[\left(\begin{array}{c}
m|\gamma| \\
m \gamma
\end{array}\right) /\left(\begin{array}{c}
k \\
\alpha
\end{array}\right)\right]^{1 /(m|\gamma|)}=\left[\frac{(m|\gamma|) ! \alpha_{1} ! \ldots \alpha_{n} !}{k !\left(m c_{1}\right) ! \ldots\left(m c_{n}\right) !}\right]^{1 /(m|\gamma|)}} \\
& \geq\left(\frac{1-\varepsilon}{1+\varepsilon}\right)^{n+1} \\
& \times \frac{m|\gamma| \cdot \alpha_{1}^{\alpha_{1} /(m|\gamma|)} \ldots \alpha_{n}^{\alpha_{n} /(m|\gamma|)} \cdot e^{k /(m|\gamma|)} \cdot e^{c_{1} /|\gamma|} \ldots e^{c_{n} /|\gamma|}}{e \cdot e^{\alpha_{1} /(m|\gamma|)} \ldots e^{\alpha_{n} /(m|\gamma|)} \cdot k^{k /(m|\gamma|)} \cdot\left(m c_{1}\right)^{c_{1} /|\gamma|} \ldots\left(m c_{n}\right)^{c_{n} /|\gamma|}} \\
& =\left(\frac{1-\varepsilon}{1+\varepsilon}\right)^{n+1}\left(\frac{\alpha_{1}}{m c_{1}}\right)^{c_{1} /|\gamma|} \ldots\left(\frac{\alpha_{n}}{m c_{n}}\right)^{c_{n} /|\gamma|} \\
& \times \alpha_{1}^{\left(\alpha_{1}-m c_{1}\right) /(m|\gamma|)} \ldots \alpha_{n}^{\left(\alpha_{n}-m c_{n}\right) /(m|\gamma|)} \cdot \frac{m|\gamma|}{k^{k /(m|\gamma|)}} \\
& \geq\left(\frac{1-\varepsilon}{1+\varepsilon}\right)^{n+1} \cdot\left(\frac{\alpha_{1}}{k}\right)^{\left(\alpha_{1}-m c_{1}\right) /(m|\gamma|)} \cdots\left(\frac{\alpha_{n}}{k}\right)^{\left(\alpha_{n}-m c_{n}\right) /(m|\gamma|)} \cdot \frac{m|\gamma|}{k} .
\end{aligned}
$$

Then

Hence

$$
L_{1} \geq\left(\frac{1-\varepsilon}{1+\varepsilon}\right)^{n+1}(1-n \varepsilon)\left(t_{1} \ldots t_{n}\right)^{\varepsilon /(1-n \varepsilon)} .
$$

$$
\begin{aligned}
r_{p}^{\prime p}(a) \geq & \left(\frac{1-\varepsilon}{1+\varepsilon}\right)^{n+1}(1-n \varepsilon)\left(t_{1} \ldots t_{n}\right)^{\varepsilon /(1-n \varepsilon)} \\
& \times K^{-n \varepsilon p /(1-n \varepsilon)} \cdot \min \left\{r_{p}^{\prime \prime p}(a),\left(r_{p}^{\prime \prime p}(a)\right)^{1 /(1-n \varepsilon)}\right\} .
\end{aligned}
$$

Since $\varepsilon$ was an arbitrary positive number, we conclude that $r_{p}^{\prime}(a) \geq r_{p}^{\prime \prime}(a)$.

Theorem 3 is proved.

We now apply the previous result to the case of $n$-tuples of operators.

Let $T=\left(T_{1}, \ldots, T_{n}\right)$ be an $n$-tuple of bounded operators in a Banach space $X$. Define

$$
\|T\|_{p}=\sup _{\substack{x \in X \\\|x\|=1}}\left(\sum_{j=1}^{n}\left\|T_{j} x\right\|^{p}\right)^{1 / p} .
$$

Equivalently, $\|T\|_{p}$ is the norm of the operator $\widetilde{T}: X \rightarrow X_{p}^{n}$, where $X_{p}^{n}$ is the direct sum of $n$ copies of $X$ endowed with the $\ell_{p}$-norm, $\left\|x_{1} \oplus \ldots \oplus x_{n}\right\|=$ $\left(\sum_{j=1}^{n}\left\|x_{j}\right\|^{p}\right)^{1 / p}$, and $\widetilde{T} x=T_{1} x \oplus \ldots \oplus T_{n} x$ (for $p=\infty$ the definitions are changed in the obvious way). Let $T=\left(T_{1}, \ldots, T_{n}\right) \in B(X)^{n}$ and $S=$ $\left(S_{1}, \ldots, S_{m}\right) \in B(X)^{m}$. Denote by $T S$ the $m n$-tuple

$$
T S=\left(T_{1} S_{1}, \ldots, T_{1} S_{m}, T_{2} S_{1}, \ldots, T_{2} S_{m}, \ldots, T_{n} S_{1}, \ldots, T_{n} S_{m}\right) .
$$


Further, let $T^{2}=T T$ and $T^{k+1}=T \cdot T^{k}$. With this notation we can state the spectral radius formula in the familiar way:

TheOREM 4. Let $T=\left(T_{1}, \ldots, T_{n}\right)$ be an $n$-tuple of mutually commuting operators in a Banach space $X$, and let $1 \leq p \leq \infty$. Then

$$
r_{p}(T)=\lim _{k \rightarrow \infty}\left\|T^{k}\right\|_{p}^{1 / k} .
$$

Proof. We have

$$
\left\|T^{k}\right\|_{p}=\sup _{\|x\|=1}\left[\sum_{|\alpha|=k}\left(\begin{array}{l}
k \\
\alpha
\end{array}\right)\left\|T^{\alpha} x\right\|^{p}\right]^{1 / p}
$$

and

$$
\begin{aligned}
r_{p}(T) & =\lim _{k \rightarrow \infty}\left[\sum_{|\alpha|=k}\left(\begin{array}{l}
k \\
\alpha
\end{array}\right)\left\|T^{\alpha}\right\|^{p}\right]^{1 /(k p)}=\lim _{k \rightarrow \infty} \max _{|\alpha|=k}\left[\left(\begin{array}{l}
k \\
\alpha
\end{array}\right)\left\|T^{\alpha}\right\|^{p}\right]^{1 /(k p)} \\
& =\lim _{k \rightarrow \infty} \max _{|\alpha|=k} \sup _{\|x\|=1}\left[\left(\begin{array}{l}
k \\
\alpha
\end{array}\right)\left\|T^{\alpha} x\right\|^{p}\right]^{1 /(k p)} \\
& =\lim _{k \rightarrow \infty} \sup _{\|x\|=1} \max _{|\alpha|=k}\left[\left(\begin{array}{l}
k \\
\alpha
\end{array}\right)\left\|T^{\alpha} x\right\|^{p}\right]^{1 /(k p)} \\
& =\lim _{k \rightarrow \infty} \sup _{\|x\|=1}\left[\sum_{|\alpha|=k}\left(\begin{array}{l}
k \\
\alpha
\end{array}\right)\left\|T^{\alpha} x\right\|^{p}\right]^{1 /(k p)}=\lim _{k \rightarrow \infty}\left\|T^{k}\right\|_{p}^{1 / k} .
\end{aligned}
$$

Remark. For $p=2$ and Hilbert space operators the previous result was proved in [6]; cf. also [3].

\section{References}

[1] M. A. Berger and Y. Wang, Bounded semigroups of matrices, Linear Algebra Appl. 166 (1992), 21-27.

[2] J. W. Bunce, Models for n-tuples of non-commuting operators, J. Funct. Anal. 57 (1984), 21-30.

[3] M. Cho and T. Huruya, On the spectral radius, Proc. Roy. Irish Acad. Sect. A 91 (1991), 39-44.

[4] M. Cho and W. Żelazko, On geometric spectral radius of commuting n-tuples of operators, Hokkaido Math. J. 21 (1992), 251-258.

[5] C.-K. Fong and A. Sołtysiak, Existence of a multiplicative linear functional and joint spectra, Studia Math. 81 (1985), 213-220.

[6] V. Müller and A. Soltysiak, Spectral radius formula for commuting Hilbert space operators, ibid. 103 (1992), 329-333.

[7] P. Rosenthal and A. Soltysiak, Formulas for the joint spectral radius of noncommuting Banach algebra elements, Proc. Amer. Math. Soc. 123 (1995), 27052708 . 
[8] G.-C. Rota and W. G. Strang, A note on the joint spectral radius, Indag. Math. 22 (1960), 379-381.

[9] A. Sołtysiak, On a certain class of subspectra, Comment. Math. Univ. Carolin. 32 (1991), 715-721.

[10] - On the joint spectral radii of commuting Banach algebra elements, Studia Math. 105 (1993), 93-99.

Mathematical Institute

Academy of Sciences of the Czech Republic

Žitná 25

11567 Praha 1, Czech Republic

E-mail: vmuller@mbox.cesnet.cz

Reçu par la Rédaction le 22.12.1994 